\title{
METHOD OF TRANSMISSION MATRIX FOR INVESTIGATING PLANAR RELATIVE MOTIONS
}

\author{
Do SANH \\ Hanoi University of Technology \\ DO DANG KHOA \\ University of Texas at Austin, USA
}

\begin{abstract}
In the paper [2] the method of transmission matrix applying for the case of a loop with turning pairs investigated. In the present paper the kinematics of a loop connected by composite joints, i.e. the one of revolute-translational joints are discussed now. By means of proposed method the planar motion of rigid bodies is presented by a point of general view.

Especially, the introduced method allows to apply effectively universal softwere, for example, MATCAD, MAPLE, ... for investigating complex mechanical systems.
\end{abstract}

\section{GENERAL INFORMATIONS ABOUT TRANSMISSION MATRIX METHOD}

Let us consider a figure $\mathrm{S}$ rotating about $O$ of the frame of reference $O x^{\prime} y^{\prime}$ and oriented by the angle $\varphi$ in counteroclockwise direction. This frame of reference is in translational displacement with respect to the fixed frame of reference ${ }^{\circ} O^{\circ} x^{o} y$. As is known, the last frame is refered to global or inertia frame of reference. An other frame of reference $O x y$ rigidly connected to the figure $S$ at its point $O$ is assigned a body frame of reference. Let us consider a point of the figure $S$. Its coordinates in these frame of reference are named global - coordinate and body-coordinate respectively. It is noticed that the body-coordinates are constants, while the global-coordinates are changing quantities at time.

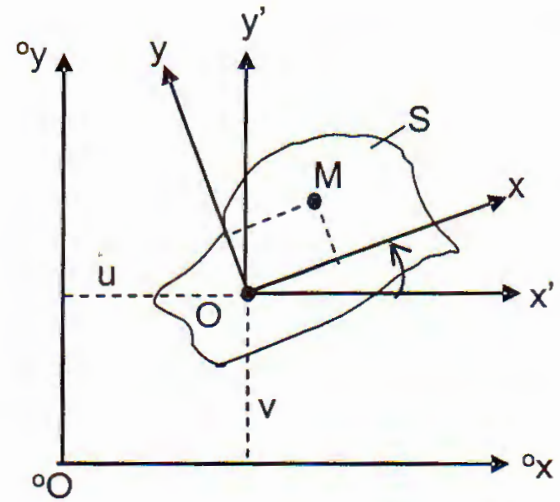

Fig. 1

Let us interest a point $M$ connected rigidly to the figure $S$. Its components of bodycoordinates are denoted by the constants $a$ and $b$. It is easy to establish the relationship between the components of global-coordinates and body-coordinates. That are

$$
{ }^{0} x_{M}=u+a \cos \varphi-b \sin \varphi ; \quad{ }^{0} y_{M}=v+a \sin \varphi+b \cos \varphi,
$$

where $u, v$ are the components of global-coordinate of the origin $O$.

The expression (1.1) can be written in the matrix form

$$
\left\|\begin{array}{c}
{ }^{0} x_{M} \\
{ }^{0} y_{M} \\
1
\end{array}\right\|=\left\|\begin{array}{lll}
1 & o & u \\
0 & 1 & 0 \\
0 & 0 & 1
\end{array}\right\|\left\|\begin{array}{lll}
1 & 0 & 0 \\
0 & 1 & v \\
0 & 0 & 1
\end{array}\right\|\left\|\begin{array}{ccc}
\cos \varphi & -\sin \varphi & 0 \\
\sin \varphi & \cos \varphi & 0 \\
0 & 0 & 1
\end{array}\right\|\left\|\begin{array}{l}
a \\
b \\
1
\end{array}\right\| .
$$


By introducing the matrices

$$
\begin{aligned}
& \mathbf{T}_{u}=\left\|\begin{array}{lll}
1 & 0 & u \\
0 & 1 & 0 \\
0 & 0 & 1
\end{array}\right\| ; \quad \mathbf{T}_{v}=\left\|\begin{array}{lll}
1 & 0 & 0 \\
0 & 1 & v \\
0 & 0 & 1
\end{array}\right\| ; \quad \mathbf{T}_{\varphi}=\left\|\begin{array}{ccc}
\cos \varphi & -\sin \varphi & 0 \\
\sin \varphi & \cos \varphi & 0 \\
0 & 0 & 1
\end{array}\right\| ; \\
& { }^{0} \mathbf{r}=\left\|\begin{array}{c}
0 \\
{ }^{0} x_{M} \\
{ }^{0} y_{M} \\
1
\end{array}\right\| ; \quad \mathbf{r}=\left\|\begin{array}{l}
a \\
b \\
1
\end{array}\right\| .
\end{aligned}
$$

The formula (1.2) takes the following form

$$
{ }^{\mathbf{0}} \mathbf{r}=\mathbf{T}_{u} \mathbf{T}_{v} \mathbf{T}_{\varphi} \mathbf{r}
$$

From now on the matrix is noted by bold letters and the vector is treated as the $(3 \times 1)$ matrix, but the frame of reference is named briefly the frame.

By (1.4) the components of the velocity of the point $M$ in the global frar $:$ is calculated by the formula

$$
{ }^{0} v=\dot{u}(*) T_{u}^{\prime} T_{v} T_{\varphi} r+\dot{v}(*) T_{u} T_{v}^{\prime} T_{\varphi} \dot{r}+\dot{\varphi}(*) T_{u} T_{v} T_{\varphi}^{\prime}(*),
$$

where [2] $\dot{\boldsymbol{u}}(*), \dot{\boldsymbol{v}}(*), \dot{\varphi}(*)$ are the $(3 \times 3)$ diagonal matrices of the form

$$
\dot{\boldsymbol{u}}(*)=\left\|\begin{array}{ccc}
\dot{u} & 0 & 0 \\
0 & \dot{u} & 0 \\
0 & 0 & \dot{u}
\end{array}\right\| ; \dot{\boldsymbol{v}}(*)=\left\|\begin{array}{ccc}
\dot{v} & 0 & 0 \\
0 & \dot{v} & 0 \\
0 & 0 & \dot{v}
\end{array}\right\| ; \dot{\varphi}(*)=\left\|\begin{array}{ccc}
\dot{\varphi} & 0 & 0 \\
0 & \dot{\varphi} & 0 \\
0 & 0 & \dot{\varphi}
\end{array}\right\|,
$$

but the matrices $T_{u}^{\prime}, T_{v}^{\prime}, T_{\varphi}^{\prime}$ are determined as follows

$$
T_{u}^{\prime}=\left\|\begin{array}{lll}
0 & 0 & 1 \\
0 & 0 & 0 \\
0 & 0 & 0
\end{array}\right\| ; T_{v}^{\prime}=\left\|\begin{array}{lll}
0 & 0 & 0 \\
0 & 0 & 1 \\
0 & 0 & 0
\end{array}\right\| ; T_{\varphi}^{\prime}=\left\|\begin{array}{ccc}
-\sin \varphi & -\cos \varphi & 0 \\
\cos \varphi & -\sin \varphi & 0 \\
0 & 0 & 0
\end{array}\right\| .
$$

Similarly, the acceleration of the point $M$ in the inertia frame is defined by the formula

$$
{ }^{0} a=\ddot{u}(*) T_{u}^{\prime} T_{v} T_{\varphi} r+\ddot{v}(*) T_{u} T_{v}^{\prime} T_{\varphi} r+\ddot{\varphi}(*) T_{u} T_{v} T_{j}^{\prime} r+\dot{\varphi}^{2}(*) T_{u} T_{v} T_{\varphi}^{\prime \prime} r
$$

where the matrices $\ddot{u}(*), \ddot{v}(*), \ddot{\varphi}(*)$ are of the same form of (1.6), in which the quantities $\dot{u}, \dot{v}, \dot{\varphi}$ are substituted by $\ddot{u}, \ddot{v}, \ddot{\varphi}$ respectively, but the matrix $T_{\varphi}^{\prime \prime}$ is of the form:

$$
T_{\varphi}^{\prime \prime}=\left\|\begin{array}{ccc}
-\cos \varphi & \sin \varphi & 0 \\
-\sin \varphi & -\cos \varphi & 0 \\
0 & 0 &
\end{array}\right\|
$$

\section{KINEMATIC INVESTIGATION OF A MULTIBODY SYSTEM}

Let us now consider a loop of $n$ links, which are connected each other by the composite joints. The links are numered in the progressive order $0,1,2, \ldots, n$, where the link of the index " 0 " is rigidly connected to the inertia frame.

For investigating such a system, let us study three of following typical groups of loops.

First group. Let us consider a loop of three bars connected together by pure turning pairs $A_{i-1}, A_{i}, A_{i+1}$ and the links are numbered by $(i-1), i$ and $(i+1)$. The body frame are chosen as follows: The $j^{\text {th }}$ body frame connected to $j^{\text {th }}$-body is denoted by $A_{j} x_{j} y_{j}$ $(j=i-1, i, i+1)$. The $x_{j}$ axis is directed from $A_{j}$ to $A_{j+1}$. The $\mathrm{y}_{j}$-axis is defined 
by following the rule of right-hand orthogonal reference frame. The angle position of the links is oriented by the angles between links, for example, the $\varphi_{i}$ is the angle between $(i-1)^{\text {th }}$-link and $i^{\text {th }}$ - link in accordance with counteroclockwise direction (Fig. 2).

Hereafter for simplicity the frames of reference are expressed by the vectors located along the $x$ axis [2]

Let us consider the point $M$ rigidly conneted to the $(i+1)^{\text {th }}$-link, which is the last one in the group. Hereafter the index located at left high corner of the quantity means that this quantity is determined with respect to the body frame of same index. For example, the point $M$ rigidly connected to the $(i+$ 1) ${ }^{\text {th }}$-body, the coordinate of which in the $(i+1)^{\text {th }}$ frame is $a$ and $b$, in the $i^{\text {th }}$-frame is ${ }^{i} x_{M},{ }^{i} y_{M}$, but

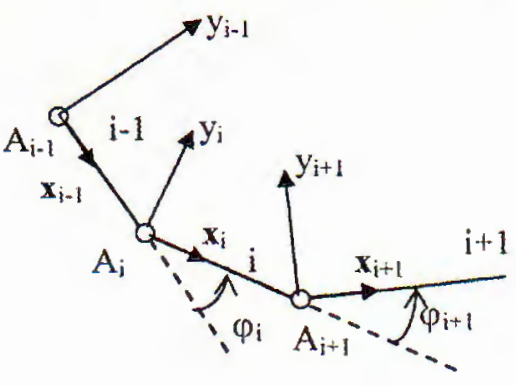

Fig. 2 in the $(i-1)^{\text {th }}$-frame is ${ }^{i-1} x_{M},{ }^{i-1} y_{M}$.

By introducing the symbols $\cos \varphi_{i} \equiv c_{i}, \sin \varphi_{i} \equiv s_{i}, L_{i-1}=\overline{A_{i-1} A_{i}} ; L_{i}=\overline{A_{i} A_{i+1}}$, where $L_{i-1}, L_{i}$ are the length of the $(i-1)^{\text {th }}$-link and the $i^{\text {th }}$-link respectively. It is noticed that $L_{i}$ is the abscissa of the origin of the $i^{\text {th }}$-body frame. It is easy to show that the configuration of the point $M$ (the position, velocity, acceleration) rigidly connected to the $(i+1)^{\text {th }}$-body frame will be

$$
\begin{aligned}
{ }^{i-1} r & =T_{i} T_{i+1} r \\
{ }^{i-1} v & =\dot{\varphi}_{i}(*) T_{i}^{\prime} T_{i+1} r+\dot{\varphi}_{i+1}(*) T_{i} T_{i+1}^{\prime} r \\
{ }^{i+1} \mathbf{a} & =\ddot{\varphi}_{i}(*) T_{i}^{\prime} T_{i+1} r+\ddot{\varphi}_{i+1}(*) T_{i} T_{i+1}^{\prime} r \\
& +\dot{\varphi}_{i}^{2}(*) T_{i}^{\prime \prime} T_{i+1} r+\dot{\varphi}_{i+1}^{2}(*) T_{i} T_{i+1}^{\prime \prime} r+2 \dot{\varphi}_{i}(*) \dot{\varphi}_{i+1}(*) T_{i}^{\prime} T_{i+1}^{\prime} r
\end{aligned}
$$

where

$$
\begin{gathered}
T_{j}=\left\|\begin{array}{ccc}
c_{j} & s_{j} & L_{j-1} \\
s_{j} & c_{j} & 0 \\
0 & 0 & 1
\end{array}\right\| ; \quad T_{j}^{\prime}=\left\|\begin{array}{ccc}
-s_{j} & -c_{j} & 0 \\
c_{j} & -s_{j} & 0 \\
0 & 0 & 0
\end{array}\right\| ; \quad T^{\prime \prime}{ }_{j}=\left\|\begin{array}{ccc}
-c_{j} & s_{j} & 0 \\
-s_{j} & -c_{j} & 0 \\
0 & 0 & 0
\end{array}\right\| \\
\dot{\varphi}_{j}(*)=\left\|\begin{array}{ccc}
\dot{\varphi}_{j} & 0 & 0 \\
0 & \dot{\varphi}_{j} & 0 \\
0 & 0 & \dot{\varphi}_{j}
\end{array}\right\| ; \quad \ddot{\varphi}_{j}(*)=\left\|\begin{array}{ccc}
\ddot{\varphi}_{j} & 0 & 0 \\
0 & \ddot{\varphi}_{j} & 0 \\
0 & 0 & \ddot{\varphi}_{j}
\end{array}\right\|
\end{gathered}
$$

\section{Second group}

Let us concern to a three bars group, in which the middle link (i.e the $i^{\text {th }}$-link) is in translational displacement with respect to the front link and connected to the behind one by revolute joint (Fig. 3). In this case the $i^{\text {th }}$-body frame is in translational displacement with respect to the $(i-1)^{\text {th }}$ body frame. Therefore the abscissa of the origin $A_{i}$ of the $i^{\text {th }}$-body frame in the $(i-1)^{\text {th }}$ body frame is not constant. In connection with this let us introduce the variable $u_{i-1} \equiv L_{i-1}$. The $x_{i-1}$ - axis coincides with the $x_{i}$-axis, but the origin of the $i^{\text {th }}$ - body frame is the one of $(i+1)^{\text {th }}$ - body frame. Because of these we have: $L_{i}=0, \varphi_{i}=0\left(c_{i}=1, s_{i}=0\right)$. 


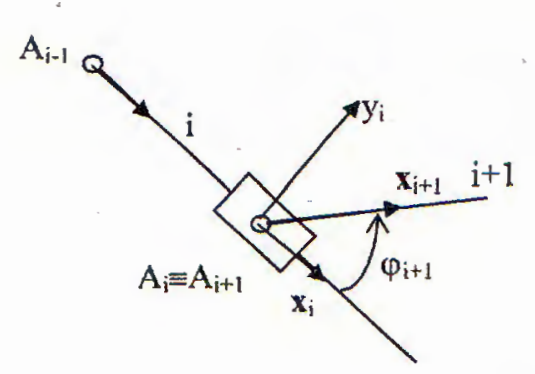

Fig. 3

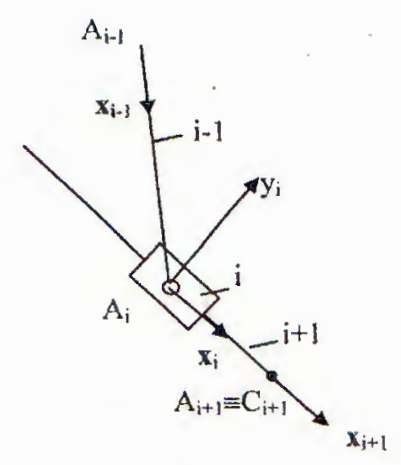

Fig. 4

In such a case we have

$$
\begin{aligned}
& T_{i}=\left\|\begin{array}{ccc}
1 & 0 & u_{i-1} \\
0 & 1 & 0 \\
0 & 0 & 0
\end{array}\right\| ; \quad T_{i}^{\prime}=\left\|\begin{array}{ccc}
0 & 0 & 1 \\
0 & 0 & 0 \\
0 & 0 & 0
\end{array}\right\| ; \quad T_{i+1}=\left\|\begin{array}{ccc}
c_{i+1} & -s_{i+1} & 0 \\
s_{i+1} & c_{i+1} & 0 \\
0 & 0 & 1
\end{array}\right\| \\
& T_{i+1}^{\prime}=\left\|\begin{array}{ccc}
-s_{i+1} & -c_{i+1} & 0 \\
c_{i+1} & -s_{i+1} & 0 \\
0 & 0 & 0
\end{array}\right\| ; \quad \mathbf{T}_{i+1}^{\prime \prime}=\left\|\begin{array}{ccc}
-c_{i+1} & s_{i+1} & 0 \\
-s_{i+1} & -c_{i+1} & 0 \\
0 & 0 & 0
\end{array}\right\|
\end{aligned}
$$

Third group

Let us consider the three bars - group in which the middle link is in translational displacement with respect to its behind link, but connected by turning pair to the front one. In this case the co-ordinate axes of the $i^{\text {th }}$-body and $(i+1)^{\text {th }}$-body frame are coincided. By this the oriented angle $\varphi_{i+1}=0\left(c_{i+1}=1, s_{i+1}=0\right)$. Because of the origin of the $(i+1)^{\text {th }}$-body frame putting at its masscenter, we have $L_{i}=\overline{A_{i} C_{i+1}}=u_{i}$, which is changing at time. The transformation matrix $T_{i+1}$ is of the form

$$
T_{i+1}=\left\|\begin{array}{ccc}
1 & 0 & u_{i} \\
0 & 1 & 0 \\
0 & 0 & 1
\end{array}\right\| .
$$

By using above mentioned models it is possible to investigate kinematics of every planar mechamism.

\section{APPLICATION}

Example 1. A quick-return mechanism is shown in Fig. 5. The crank $O A=r$ at given instant of time is in horizontal line on the right side at the angular velocity and acceleration $\omega_{1}, \varepsilon_{1}$ respectively. The distance between two rotating axes $O C=h$. Determine the angular velocity and acceleration of the crank $C B$ at the interested position of the mechanism.

The mechanism consists of three links numered by the order $1,2,3$. The inertia frame is expressed by the vector $x_{o}$ putting at $O$ and being in the horizontal direction.

The body frames are represented the vectors $x_{1}, x_{2}, x_{3}$ as shown in Fig. 5 . The origin of these vectors located $O, A$ and $B$, respectively. 


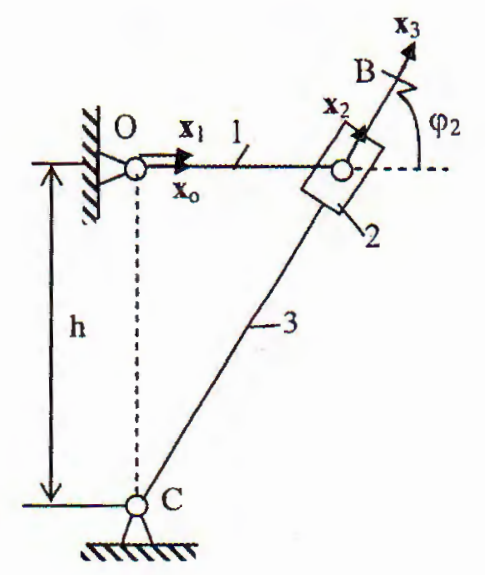

Fig. 5

In accordance with above mentioned things we have:

$$
\varphi_{1}=0\left(c_{1}=1, s_{1}=0\right) ; \quad \varphi_{3}=0\left(c_{3}=1, s_{3}=0\right) ; \quad L_{2}=\overline{A B}=u
$$

where $u$ is a changing quantity at time.

The mechanism under consideration belonging to the third group is a closed loop. in connection with the latter it is necessary to interest the point $C$, which is fixed in the global frame with the coordinates $(0,-h)$. The body-coordinates of the point $C$ are $(-L, 0)$.

By applying (2.1) in consideration of (2.6) and $L_{1}=r$, we get

$$
\left\|\begin{array}{c}
0 \\
-h \\
1
\end{array}\right\|=\left\|\begin{array}{lll}
1 & 0 & 0 \\
0 & 1 & 0 \\
0 & 0 & 1
\end{array}\right\|\left\|\begin{array}{ccc}
c_{2} & -s_{2} & r \\
s_{2} & c_{2} & 0 \\
0 & 0 & 1
\end{array}\right\|\left\|\begin{array}{ccc}
1 & 0 & u \\
0 & 1 & 0 \\
0 & 0 & 1
\end{array}\right\|\|\| \begin{gathered}
-L \\
0 \\
1
\end{gathered}\|=\| \begin{gathered}
{\left[c_{2}(u-L)+r\right]} \\
s_{2}(u-L) \\
1
\end{gathered} \|
$$

In accordance with the given position, $L-u=\overline{C A}=\sqrt{r^{2}+h^{2}}$. By solving the obtained matrix equation, it is easy to calculate

$$
c_{2}^{*}=\frac{r}{\sqrt{r^{2}+h^{2}}} ; \quad s_{2}^{*}=\frac{h}{\sqrt{r^{2}+h^{2}}}
$$

where the symbol " *" is the notation of the value of the quantity at the given instant of time.

In order to calculate the angular velocity of the crank $C B$ let us write the equations (1.5) in consideration of (2.4) and (2.6), that is

$$
\left\|\begin{array}{l}
0 \\
0 \\
0
\end{array}\right\|=\dot{\varphi}_{1}(*)\left\|\begin{array}{ccc}
0 & -1 & 0 \\
1 & 0 & 0 \\
0 & 0 & 0
\end{array}\right\|\left\|\begin{array}{ccc}
c_{2}^{*} & -s_{2}^{*} & r \\
s_{2}^{*} & c_{2}^{*} & 0 \\
0 & 0 & 1
\end{array}\right\|\left\|\begin{array}{ccc}
1 & 0 & u \\
0 & 1 & 0 \\
0 & 0 & 1
\end{array}\right\|\left\|\begin{array}{c}
-L \\
0 \\
1
\end{array}\right\|
$$




$$
\begin{aligned}
& +\dot{\varphi}_{2}(*)\left\|\begin{array}{ccc}
1 & 0 & 0 \\
0 & 1 & 0 \\
0 & 0 & 1
\end{array}\right\|\left\|\begin{array}{ccc}
-s_{2}^{*} & -c_{2}^{*} & 0 \\
c_{2}^{*} & -s_{2}^{*} & 0 \\
0 & 0 & 0
\end{array}\right\|\|\| \begin{array}{c}
-L \\
0 \\
1
\end{array} \\
& +\dot{u}(*)\left\|\begin{array}{ccc}
1 & 0 & 0 \\
0 & 1 & 0 \\
0 & 0 & 1
\end{array}\right\|\left\|\begin{array}{ccc}
c_{2}^{*} & -s_{2}^{*} & r \\
s_{2}^{*} & c_{2}^{*} & 0 \\
0 & 0 & 1
\end{array}\right\|\left\|\begin{array}{ccc}
0 & 0 & 1 \\
0 & 0 & 0 \\
0 & 0 & 0
\end{array}\right\|\left\|\begin{array}{c}
-L \\
0 \\
1
\end{array}\right\| .
\end{aligned}
$$

By developing the written equation, we get

$$
\begin{aligned}
& s_{2}^{*}\left(L-u^{*}\right) \dot{\varphi}_{1}^{*}+s_{2}^{*}\left(L-u^{*}\right) \dot{\varphi}_{2}^{*}+c_{2}^{*} \dot{u}^{*}=0 ; \\
& c_{2}^{*}\left(L-u^{*}+r\right) \dot{\varphi}_{1}^{*}+c_{2}^{*}\left(L-u^{*}\right) \dot{\varphi}_{2}^{*}+s_{2}^{*} \dot{u}^{*}=0 .
\end{aligned}
$$

The solutions of these equations are of the form

$$
\dot{\varphi}_{2}^{*}=-\dot{\varphi}_{1}^{*}+\frac{c_{2}^{*}}{\left(L-u^{*}\right)} r \dot{\varphi}_{1}^{*} ; \quad \dot{u}^{*}=-r s_{2}^{*} \dot{\varphi}_{1}^{*}
$$

The absolute angular velocity of the crank $C B$ denoted by $\omega_{2}$ will be [2]

$$
\omega_{2}=\dot{\varphi}_{1}^{*}+\dot{\varphi}_{2}^{*}=\frac{c_{2}^{*}}{\left(L-u^{*}\right)} r \dot{\varphi}_{1}^{*}=\frac{r}{\sqrt{r^{2}+h^{2}}} c_{2}^{*} \omega_{1} .
$$

The relative velocity of the slider $A$ with respect to the bar $C B$ at the given instant of time is of the form

$$
v_{r}^{*}=-\dot{u}^{*}=r s_{2}^{*} \dot{\varphi}_{1}^{*}=r \omega_{1} s_{2}^{*} .
$$

The positive signal of the expression of the velocity provides that the relative velocity the same direction to the vector $x_{3}$.

In other words, at the given instant of time the relative velocity is in the direction from $A$ to $B$.

In order to define the acceleration, let us apply the formula (2.3) in consideration of (2.4) and (2.6), that are

$$
\begin{aligned}
& \left\|\begin{array}{l}
0 \\
0 \\
0
\end{array}\right\|=\ddot{\varphi}_{1}(*)\left\|\begin{array}{ccc}
0 & -1 & 0 \\
1 & 0 & 0 \\
0 & 0 & 0
\end{array}\right\|\left\|\begin{array}{ccc}
c_{2}^{*} & -s_{2}^{*} & r \\
s_{2}^{*} & c_{2}^{*} & 0 \\
0 & 0 & 1
\end{array}\right\|\left\|\begin{array}{ccc}
1 & 0 & u^{*} \\
0 & 1 & 0 \\
0 & 0 & 1
\end{array}\right\|\left\|\begin{array}{c}
-L \\
0 \\
1
\end{array}\right\| \\
& +\ddot{\varphi}_{2}(*)\left\|\begin{array}{ccc}
1 & 0 & 0 \\
0 & 1 & 0 \\
0 & 0 & 1
\end{array}\right\|\left\|\begin{array}{ccc}
-s_{2}^{*} & -c_{2}^{*} & 0 \\
c_{2}^{*} & -s_{2}^{*} & 0 \\
0 & 0 & 0
\end{array}\right\|\left\|\begin{array}{c}
-L \\
0 \\
1
\end{array}\right\| \\
& +\ddot{u}(*)\left\|\begin{array}{ccc}
1 & 0 & 0 \\
0 & 1 & 0 \\
0 & 0 & 1
\end{array}\right\|\left\|\begin{array}{ccc}
c_{2}^{*} & -s_{2}^{*} & r \\
s_{2}^{*} & c_{2}^{*} & 0 \\
0 & 0 & 1
\end{array}\right\|\left\|\begin{array}{ccc}
0 & 0 & 1 \\
0 & 0 & 0 \\
0 & 0 & 0
\end{array}\right\|\left\|\begin{array}{c}
-L \\
0 \\
1
\end{array}\right\| \\
& +\dot{\varphi}_{1}^{* 2}(*)\left\|\begin{array}{ccc}
-1 & 0 & 0 \\
0 & -1 & 0 \\
0 & 0 & 0
\end{array}\right\|\left\|\begin{array}{ccc}
c_{2}^{*} & -s_{2}^{*} & r \\
s_{2}^{*} & c_{2}^{*} & 0 \\
0 & 0 & 1
\end{array}\right\|\|\| \begin{array}{ccc}
1 & 0 & u^{*} \\
0 & 1 & 0 \\
0 & 0 & 1
\end{array}\|\|\left\|\begin{array}{c}
-L \\
0 \\
1
\end{array}\right\|
\end{aligned}
$$




$$
\begin{aligned}
& +\dot{\varphi}_{2}^{* 2}\left\|\begin{array}{ccc}
1 & 0 & 0 \\
0 & 1 & 0 \\
0 & 0 & 1
\end{array}\right\|\left\|\begin{array}{ccc}
-s_{2}^{*} & -c_{2}^{*} & 0 \\
c_{2}^{*} & -s_{2}^{*} & 0 \\
0 & 0 & 0
\end{array}\right\|\left\|\begin{array}{|ccc}
1 & 0 & u^{*} \\
0 & 1 & 0 \\
0 & 0 & 1
\end{array}\right\|\left\|\begin{array}{c}
-L \\
0 \\
1
\end{array}\right\| \\
& +2 \dot{\varphi}_{1}^{*}(*) \dot{\varphi}_{2}^{*}(*)\left\|\begin{array}{ccc}
0 & -1 & 0 \\
1 & 0 & 0 \\
0 & 0 & 0
\end{array}\right\|\left\|\begin{array}{ccc}
-s_{2}^{*} & -c_{2}^{*} & 0 \\
c_{2}^{*} & -s_{2}^{*} & 0 \\
0 & 0 & 0
\end{array}\right\|\left\|\begin{array}{ccc}
1 & 0 & u^{*} \\
0 & 1 & 0 \\
0 & 0 & 1
\end{array} \mid\right\| \| \begin{array}{c}
-L \\
0 \\
1
\end{array} \\
& +2 \dot{\varphi}_{1}^{*}(*) \dot{\mathbf{u}}^{*}(*)\left\|\begin{array}{ccc}
0 & -1 & 0 \\
1 & 0 & 0 \\
0 & 0 & 0
\end{array}\right\|\left\|\begin{array}{ccc}
c_{2}^{*} & -s_{2}^{*} & r \\
s_{2}^{*} & c_{2}^{*} & 0 \\
0 & 0 & 1
\end{array}\right\| \| \begin{array}{c}
-L \\
0 \\
1
\end{array} \\
& +2 \dot{\varphi}_{2}^{*}(*) \dot{\mathbf{u}}^{*}(*)\left\|\begin{array}{ccc}
1 & -0 & 0 \\
0 & 1 & 0 \\
0 & 0 & 1
\end{array}\right\|\left\|\begin{array}{ccc}
-s_{2}^{*} & -c_{2}^{*} & 0 \\
c_{2}^{*} & -s_{2}^{*} & 0 \\
0 & 0 & 0
\end{array}\right\|\left\|\begin{array}{c}
-L \\
0 \\
1
\end{array}\right\| .
\end{aligned}
$$

By solving above establised matrix equation we obtain

$$
\begin{aligned}
& \ddot{\varphi}_{2}^{*}=\left(\frac{c_{2}^{*} r}{L-u^{*}}-1\right) \ddot{\varphi}_{1}^{*}+\frac{1}{L-u^{*}}\left[r s_{2}^{*} \dot{\varphi}_{1}^{* 2}-2 \dot{\varphi}_{1}^{*}+\dot{\varphi}_{2}^{*}\right] \dot{u}^{*} ; \\
& \ddot{u}^{*}=-\left(L-u^{*}\right)\left(\dot{\varphi}_{1}^{*}+\dot{\varphi}_{2}^{*}\right)^{2}+r \dot{\varphi}_{1}^{* 2} c_{2}^{*} .
\end{aligned}
$$

In consideration of

$$
\begin{array}{lll}
\dot{\varphi}_{1}^{*} \equiv \omega_{1}, \quad \dot{\varphi}_{1}^{*}+\dot{\varphi}_{2}^{*} \equiv \omega_{2}, \quad \ddot{\varphi}_{1} \equiv \varepsilon_{1} ; & \ddot{\varphi}_{1}+\ddot{\varphi}_{2}=\varepsilon_{2} ; \\
L-u^{*}=\sqrt{r^{2}+h^{2}} ; \quad s_{2}^{*}=\frac{h}{\sqrt{r^{2}+h^{2}}} ; & c_{2}^{*}=\frac{r}{\sqrt{r^{2}+h^{2}}},
\end{array}
$$

we get

$$
\varepsilon_{2}=\ddot{\varphi}_{1}^{*}+\ddot{\varphi}_{2}^{*}=\frac{r^{2}}{r^{2}+h^{2}} \varepsilon_{1}+\frac{r h}{r^{2}+h^{2}} \omega_{1}^{2}-\frac{2}{\sqrt{r^{2}+h^{2}}} \dot{u}^{*} \omega_{2},
$$

where: $\omega_{1}, \omega_{2}, \varepsilon_{1}, \varepsilon_{2}$ are the absolute angular velocities and accelerations of the bars $O A$ and $C B$ respectively

Example 2. Let us consider a mechanism shown in Fig. 6. The length of the rod $A C=L$. The vertical guider located at the distance $h$ with respect to the fixed rotating axis $O$. Determine the velocity and acceleration of the sliding rod $C A$

The mechanism under consideration consists of three bars, numered in the order $1,2,3$, in which the slider $A$ (link 2) is in translational displacement with respect to the crank 1. By such a way the consider mechanism belongs second group. The inertia frame is expressed by the $\mathbf{x}_{o}$ in horizontal direction. The body frames are expressed by the vectors $\boldsymbol{x}_{1}, \boldsymbol{x}_{2}, \boldsymbol{x}_{3}$ respectively as in Fig. 6. The origin $A$ of the $2^{\text {nd }}$ body frame displaces along the axis of the $x$-axis of the $1^{\text {st }}$ - body frame. In accordance with this we have

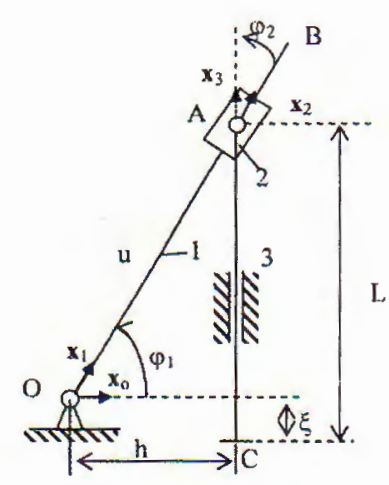

Fig. 6

$$
\varphi_{2}=0 \rightarrow c_{2}^{*}=1, s_{2}^{*}=0 .
$$


In addition, the oriented angle of the bar $C A$ in the global frame is equal to Therefore:

$$
\varphi_{1}+\varphi_{2}+\varphi_{3}=\frac{\pi}{2} \rightarrow \varphi_{1}+\varphi_{3}=\frac{\pi}{2} \rightarrow s_{3}=c_{1}, \quad c_{3}=s_{1} .
$$

Besides, the mechanism under consideration is a semi-closed loop, because the point $C$ of the last link (link 3) is in displacement along the guider fixed in the global frame. The global and body coordinates of the point $C$ are $(h,-\xi)$ and $(-L, 0)$ respectively.

By applying (2.1) in consideration of (2.5) we get

$$
\left[\begin{array}{c}
h \\
-\xi \\
1
\end{array}\right]=\left\|\begin{array}{ccc}
c_{1}^{*} & -s_{1}^{*} & 0 \\
s_{1}^{*} & c_{1}^{*} & 0 \\
0 & 0 & 1
\end{array}\right\|\left\|\begin{array}{ccc}
1 & 0 & u \\
0 & 1 & 0 \\
0 & 0 & 1
\end{array}\right\|\left\|\begin{array}{ccc}
s_{1}^{*} & -c_{1}^{*} & 0 \\
c_{1}^{*} & s_{1}^{*} & 0 \\
0 & 0 & 1
\end{array}\right\|\left\|\begin{array}{c}
-L \\
0 \\
1
\end{array}\right\|
$$

By the establised matrix equation, we find:

$$
s_{3}^{*}=\frac{h}{u^{*}} ; \quad c_{3}^{*}=\frac{L-\xi^{*}}{u^{*}} .
$$

In order to calculate the velocity, let us write the equation (2.2), which is of the form

$$
\begin{aligned}
\left\|\begin{array}{c}
0 \\
\dot{\xi}^{*} \\
0
\end{array}\right\|= & \dot{\varphi}_{1}(*)\left\|\begin{array}{ccc}
-s_{1}^{*} & -c_{1}^{*} & 0 \\
c_{1}^{*} & -s_{1}^{*} & 0 \\
0 & 0 & 1
\end{array}\right\|\|\| \begin{array}{ccc}
1 & 0 & u^{*} \\
0 & 1 & 0 \\
0 & 0 & 1
\end{array}\|\| \begin{array}{ccc}
s_{1}^{*} & -c_{1}^{*} & 0 \\
c_{1}^{*} & s_{1}^{*} & 0 \\
0 & 0 & 1
\end{array}\|\| \| \begin{array}{c}
-L \\
0 \\
1
\end{array} \\
& +i_{2}(*)\left\|\begin{array}{ccc}
c_{1}^{*} & -s_{1}^{*} & 0 \\
s_{1}^{*} & c_{1}^{*} & 0 \\
0 & 0 & 1
\end{array}\right\|\left\|\begin{array}{ccc}
0 & 0 & 1 \\
0 & 0 & 0 \\
0 & 0 & 0
\end{array}\right\|\left\|\begin{array}{ccc}
s_{1}^{*} & -c_{1}^{*} & 0 \\
c_{1}^{*} & s_{1}^{*} & 0 \\
0 & 0 & 1
\end{array}\right\| \| \begin{array}{c}
-L \\
0 \\
1
\end{array} \\
& +\dot{\varphi}_{3}^{*}(*)\left\|\begin{array}{ccc}
c_{1}^{*} & -s_{1}^{*} & 0 \\
s_{1}^{*} & c_{1}^{*} & 0 \\
0 & 0 & 1
\end{array}\right\|\left\|\begin{array}{ccc}
1 & 0 & u^{*} \\
0 & 1 & 0 \\
0 & 0 & 1
\end{array}\right\|\left\|\begin{array}{ccc}
c_{1}^{*} & s_{1}^{*} & 0 \\
-s_{1}^{*} & c_{1}^{*} & 0 \\
0 & 0 & 0
\end{array}\right\|\|\| \begin{array}{c}
-L \\
0 \\
1
\end{array} \| .
\end{aligned}
$$

By this equation we calculate

$$
\dot{\xi}^{*}=\frac{s_{1}^{*}}{c_{1}^{*}} u^{*} \varphi_{1}^{*}=\frac{s_{1}^{*}}{c_{1}^{*}} u^{*} \omega_{1} ; \quad \dot{u}^{*}=c_{1}^{*} u^{*} \dot{\varphi}_{1}^{*}=c_{1}^{*} u^{*} \omega_{1} .
$$

Concerning to the problem of acceleration, let us write the equation (2.3), that is

$$
\begin{aligned}
& \left\|\begin{array}{l}
0 \\
\ddot{\xi}^{*} \\
0
\end{array}\right\|=\ddot{\varphi}_{1}^{*}(*)\left\|\begin{array}{ccc}
-s_{1}^{*} & -c_{1}^{*} & 0 \\
c_{1}^{*} & -s_{1}^{*} & 0 \\
0 & 0 & 1
\end{array}\right\|\left\|\begin{array}{ccc}
1 & 0 & u \\
0 & 1 & 0 \\
0 & 0 & 1
\end{array}\right\|\left\|\begin{array}{ccc}
s_{1}^{*} & -c_{1}^{*} & 0 \\
c_{1}^{*} & s_{1}^{*} & 0 \\
0 & 0 & 1
\end{array}\right\| \| \mid \begin{array}{c}
-L \\
0 \\
1
\end{array} \\
& +\ddot{\mathbf{u}}(*)\left\|\begin{array}{ccc}
c_{1}^{*} & -s_{1}^{*} & 0 \\
s_{1}^{*} & c_{1}^{*} & 0 \\
0 & 0 & 1
\end{array}\right\|\left\|\begin{array}{ccc}
0 & 0 & 1 \\
0 & 0 & 0 \\
0 & 0 & 0
\end{array}\right\|\left\|\begin{array}{ccc}
s_{1}^{*} & -c_{1}^{*} & 0 \\
c_{1}^{*} & s_{1}^{*} & 0 \\
0 & 0 & 1
\end{array}\right\|\left\|\begin{array}{c}
-L \\
0 \\
1
\end{array}\right\| \\
& +\ddot{\varphi}_{3}^{*}(*)\left\|\begin{array}{ccc}
c_{1}^{*} & -s_{1}^{*} & 0 \\
s_{1}^{*} & c_{1}^{*} & 0 \\
0 & 0 & 1
\end{array}\right\|\left\|\begin{array}{ccc}
1 & 0 & u^{*} \\
0 & 1 & 0 \\
0 & 0 & 1
\end{array}\right\|\left\|\begin{array}{ccc}
-c_{1}^{*} & -s_{1}^{*} & 0 \\
s_{1}^{*} & -c_{1}^{*} & 0 \\
0 & 0 & 0
\end{array}\right\|\|\| \begin{array}{c}
-L \\
0 \\
1
\end{array}
\end{aligned}
$$




$$
\begin{aligned}
& +\dot{\varphi}_{1}^{* 2}(*)\left\|\begin{array}{ccc}
-c_{1}^{*} & s_{1}^{*} & 0 \\
-s_{1}^{*} & -c_{1}^{*} & 0 \\
0 & 0 & 0
\end{array}|| \begin{array}{|lll}
1 & 0 & u^{*} \\
0 & 1 & 0 \\
0 & 0 & 1
\end{array}\right\|\left\|\begin{array}{ccc}
s_{1}^{*} & -c_{1}^{*} & 0 \\
c_{1}^{*} & s_{1}^{*} & 0 \\
0 & 0 & 1
\end{array}\right\| \| \begin{array}{c}
-L \\
0 \\
1
\end{array} \\
& +\dot{\varphi}_{3}^{* 2}(*)\left\|\begin{array}{ccc}
c_{1}^{*} & -s_{1}^{*} & 0 \\
s_{1}^{*} & c_{1}^{*} & 0 \\
0 & 0 & 1
\end{array}\right\|\left\|\begin{array}{ccc}
1 & 0 & u^{*} \\
0 & 1 & 0 \\
0 & 0 & 1
\end{array}\right\|\left\|\begin{array}{ccc}
-s_{1}^{*} & c_{1}^{*} & 0 \\
-c_{1}^{*} & -s_{1}^{*} & 0 \\
0 & 0 & 0
\end{array}\right\| \| \begin{array}{c}
-L \\
0 \\
1
\end{array} \\
& +2 \dot{\boldsymbol{\varphi}}_{1}^{*}(*) \dot{\mathbf{u}}^{*}(*)\left\|\begin{array} { c c c } 
{ - s _ { 1 } ^ { * } } & { - c _ { 1 } ^ { * } } & { 0 } \\
{ c _ { 1 } ^ { * } } & { - s _ { 1 } ^ { * } } & { 0 } \\
{ 0 } & { 0 } & { 1 }
\end{array} \left|\left\|\mid \begin{array}{ccc}
0 & 0 & 1 \\
0 & 0 & 0 \\
0 & 0 & 0
\end{array}\right\|\left\|\begin{array}{ccc}
s_{1}^{*} & -c_{1}^{*} & 0 \\
c_{1}^{*} & s_{1}^{*} & 0 \\
0 & 0 & 1
\end{array}\right\|\|\| \begin{array}{c}
-L \\
0 \\
1
\end{array}\right.\right. \\
& +2 \dot{\varphi}_{1}^{*}(*) \dot{\varphi}_{3}^{*}(*)\left\|\begin{array}{ccc}
-s_{1}^{*} & -c_{1}^{*} & 0 \\
c_{1}^{*} & -s_{1}^{*} & 0 \\
0 & 0 & 0
\end{array}\right\|\left\|\begin{array}{ccc}
1 & 0 & u^{*} \\
0 & 1 & 0 \\
0 & 0 & 1
\end{array}\right\|\left\|\begin{array}{ccc}
-c_{1}^{*} & -s_{1}^{*} & 0 \\
s_{1}^{*} & -c_{1}^{*} & 0 \\
0 & 0 & 0
\end{array}\right\| \| \begin{array}{c}
-L \\
0 \\
1
\end{array} \\
& +2 \dot{\varphi}_{\mathbf{3}}^{*}(*) \dot{\mathbf{u}}^{*}(*)\left\|\begin{array}{ccc}
c_{1}^{*} & -s_{1}^{*} & 0 \\
s_{1}^{*} & c_{1}^{*} & 0 \\
0 & 0 & 1
\end{array}\right\|\left\|\begin{array}{ccc}
0 & 0 & 1 \\
0 & 0 & 0 \\
0 & 0 & 0
\end{array}\right\|\left\|\begin{array}{ccc}
-c_{1}^{*} & -s_{1}^{*} & 0 \\
s_{1}^{*} & -c_{1}^{*} & 0 \\
0 & 0 & 0
\end{array}\right\|\left\|\begin{array}{c}
-L \\
0 \\
1
\end{array}\right\|
\end{aligned}
$$

By realizing the calculations we find

$$
\begin{aligned}
& \ddot{u}=\frac{s_{1}^{*}}{c_{1}^{*}} u^{*} \ddot{\varphi}_{1}^{*}+u^{*} \dot{\varphi}_{1}^{* 2}+2 \frac{s_{1}^{*}}{c_{1}^{*}} u^{*} \dot{\varphi}_{1}^{* 2}=\frac{s_{1}^{*}}{c_{1}^{*}} u^{*} \varepsilon_{1}+u^{*} \omega_{1}^{2} ; \\
& \ddot{\xi}=\frac{\left(u^{*} \ddot{\varphi}_{1}^{*}+2 \dot{u}^{*} \dot{\varphi}_{1}^{*}\right)}{c_{1}^{*}} \equiv \frac{\left(u^{*} \varepsilon_{1}+2 \dot{u}^{*} \omega_{1}\right)}{c_{1}^{*}} .
\end{aligned}
$$

Example 3. The crank $O A$, turning about fixed $O$, is connected by turning pair to link 2 , which moves in slider 3 . Slider 3 turns about fixed axis $O$. Determine the angular velocity and acceleration of the link2 when the crank $O A$ is invertical direction (Fig. 7).

The mechanism under consideration is a closed three bars loop, because the point $C$ of the last link of the group (link 3) is fixed in the inertia frame, which is expressed by the vector $x_{0}$. The body frame are expressed by the vectors $x_{1}, x_{2}, x_{3}$ shown as in Fig. 7 .

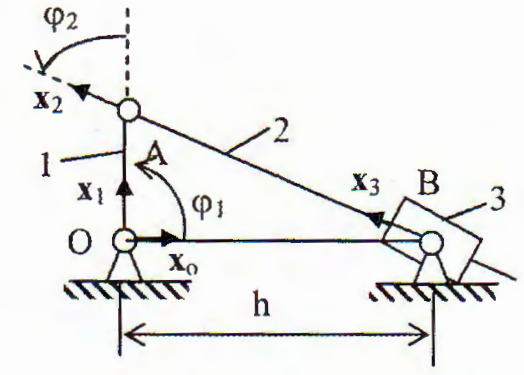

Fig. 7

The link 2 is in the translational displacement with respect to the last link (link 3). The mechanism belongs to third group. In connection with this, it is easy to see that $\varphi_{3}^{*}=0 \rightarrow c_{3}^{*}=1, s_{3}^{*}=0$. Besides, the origin of $3^{\text {th }}$-body frame is in displacement with respect to $2^{\text {nd }}$ body frame, i.e. the quantity $L_{2}=u$ is variable. The global and body coordinate of the point $\mathrm{B}$ belonging to the link 3 are $(h, 0)$ and $(0,0)$ respectively. Applying the formula (2.1) for this point, we obtain the equation for defining the position of the mechanism at given time, that is

$$
\left\|\begin{array}{l}
h \\
0 \\
1
\end{array}\right\|=\left\|\begin{array}{ccc}
0 & -1 & 0 \\
1 & 0 & 0 \\
0 & 0 & 1
\end{array}\right\|\left\|\begin{array}{ccc}
c_{2}^{*} & -s_{2}^{*} & r \\
s_{2}^{*} & c_{2}^{*} & 0 \\
0 & 0 & 1
\end{array}\right\|\left\|\begin{array}{ccc}
1 & 0 & -u \\
0 & 1 & 0 \\
0 & 0 & 1
\end{array}\right\|\left\|\begin{array}{l}
0 \\
0 \\
1
\end{array}\right\|
$$

By means of solving this equation, we find 


$$
s_{2}^{*}=\frac{h}{u^{*}} ; \quad c_{2}^{*}=\frac{r}{u^{*}} .
$$

In order to determine the velocity let us write the equation (2.2) for the point $B$, that is

$$
\begin{aligned}
& \left\|\begin{array}{l}
0 \\
0 \\
0
\end{array}\right\|=\dot{\varphi}_{1}^{*}(*)\left\|\begin{array}{ccc}
-1 & 0 & 0 \\
0 & 1 & 0 \\
0 & 0 & 1
\end{array}\right\|\left\|\begin{array}{ccc}
c_{2}^{*} & -s_{2}^{*} & r \\
s_{2}^{*} & c_{2}^{*} & 0 \\
0 & 0 & 1
\end{array}\right\|\left\|\begin{array}{ccc}
1 & 0 & -u^{*} \\
0 & 1 & 0 \\
0 & 0 & 1
\end{array}\right\|\left\|\begin{array}{c}
0 \\
0 \\
1
\end{array}\right\| \\
& +\dot{\varphi}_{2}^{*}(*)\left\|\begin{array}{ccc}
0 & -1 & 0 \\
1 & 0 & 0 \\
0 & 0 & 1
\end{array}\right\|\left\|\begin{array} { c c c } 
{ - s _ { 2 } ^ { * } } & { - c _ { 2 } ^ { * } } & { 0 } \\
{ c _ { 2 } ^ { * } } & { - s _ { 2 } ^ { * } } & { 0 } \\
{ 0 } & { 0 } & { 0 }
\end{array} \left|\left\|\mid \begin{array}{ccc}
1 & 0 & -u^{*} \\
0 & 1 & 0 \\
0 & 0 & 1
\end{array}\right\|\left\|\begin{array}{|l}
0 \\
0 \\
1
\end{array}\right\|\right.\right. \\
& +\dot{\boldsymbol{u}}^{*}(*)\left\|\begin{array}{ccc}
0 & -1 & 0 \\
1 & 0 & 0 \\
0 & 0 & 1
\end{array}\right\|\left\|\begin{array}{ccc}
c_{2}^{*} & -s_{2}^{*} & r \\
s_{2}^{*} & c_{2}^{*} & 0 \\
0 & 0 & 1
\end{array}\right\|\left\|\begin{array}{ccc}
0 & 0 & -1 \\
0 & 0 & 0 \\
0 & 0 & 0
\end{array}\right\|\left\|\begin{array}{|l}
0 \\
0 \\
1
\end{array}\right\| \\
& =\left\|\begin{array}{c}
{\left[s_{2}^{*} \dot{u}^{*}+c_{2}^{*} u^{*} \dot{\varphi}_{2}^{*}-\left(c_{2}^{*} u^{*}-r\right) \dot{\varphi}_{1}^{*}\right]} \\
{\left[c_{2}^{*} \dot{u}^{*}-s_{2}^{*} u^{*} \dot{\varphi}_{2}^{*}-s_{2}^{*} u^{*} \dot{\varphi}_{1}^{*}\right]} \\
1
\end{array}\right\|
\end{aligned}
$$

It is easy to obtain

$$
\dot{u}^{*}=s_{2}^{*} r \dot{\varphi}_{1}^{*}=s_{2}^{*} r \omega_{1} ; \quad \dot{\varphi}_{2}^{*}=-\dot{\varphi}_{1}^{*}+\frac{c_{2}^{*}}{u^{*}} r \dot{\varphi}_{1}^{*}
$$

The absolute angular velocity of the sliding $\operatorname{rod} A B$ will be

$$
\omega_{2}=\dot{\varphi}_{1}^{*}+\dot{\varphi}_{2}^{*}=\frac{r^{2}}{u^{* 2}} \omega_{1}^{2}
$$

The relative velocity of the pitton $B$ with respect to the sliding $\operatorname{rod} A B$ is equal to

$$
v_{e}=-u^{*}-s_{2}^{*} r \dot{\varphi}_{1}^{*}=-\frac{h}{u^{*}} r \omega_{1}
$$

For the aim of calculating acceleration, let us write equation (2.3) for the point $B$, that is

$$
\begin{aligned}
& \left\|\begin{array}{l}
0 \\
0 \\
0
\end{array}\right\|=\ddot{\varphi}_{1}^{*}(*)\left\|\begin{array}{ccc}
-1 & 0 & 0 \\
0 & 1 & 0 \\
0 & 0 & 1
\end{array}\right\|\left\|\begin{array}{ccc}
c_{2}^{*} & -s_{2}^{*} & r \\
s_{2}^{*} & c_{2}^{*} & 0 \\
0 & 0 & 1
\end{array}\right\|\left\|\begin{array}{ccc}
1 & 0 & -u^{*} \\
0 & 1 & 0 \\
0 & 0 & 1
\end{array} \mid\right\| \begin{array}{l}
0 \\
0 \\
1
\end{array} \| \\
& +\ddot{\varphi}_{2}^{*}(*)\left\|\begin{array}{ccc}
0 & -1 & 0 \\
1 & 0 & 0 \\
0 & 0 & 0
\end{array}\right\|\left\|\begin{array}{ccc}
-s_{2}^{*} & -c_{2}^{*} & 0 \\
c_{2}^{*} & -s_{2}^{*} & 0 \\
0 & 0 & 0
\end{array}\right\| \\
& +\ddot{\mathbf{u}}^{*}(*)\left\|\begin{array}{ccc}
0 & -1 & 0 \\
1 & 0 & 0 \\
0 & 0 & 1
\end{array}\right\|\left\|\begin{array}{ccc||ccc}
c_{2}^{*} & -s_{2}^{*} & r \\
s_{2}^{*} & c_{2}^{*} & 0 \\
0 & 0 & 1
\end{array}\right\| \mid \begin{array}{ccc}
0 & 0 & -1 \\
0 & 0 & 0 \\
0 & 0 & 0
\end{array}\|\| \begin{array}{c}
0 \\
0 \\
1
\end{array} \|
\end{aligned}
$$




$$
\begin{aligned}
& +\dot{\varphi}_{1}^{* 2}(*)\left\|\begin{array}{ccc}
0 & 1 & 0 \\
-1 & 0 & 0 \\
0 & 0 & 1
\end{array}\right\|\left\|\begin{array}{ccc}
c_{2}^{*} & -s_{2}^{*} & r \\
-s_{2}^{*} & c_{2}^{*} & 0 \\
0 & 0 & 1
\end{array}\left|\left\|\left|\begin{array}{ccc}
1 & 0 & -u^{*} \\
0 & 1 & 0 \\
0 & 0 & 1
\end{array}\right|\right\|\right| \mid \begin{array}{l}
0 \\
0 \\
1
\end{array}\right\| \\
& +\dot{\varphi}_{2}^{* 2}(*)\left\|\begin{array}{ccc}
0 & 1 & 0 \\
1 & 0 & 0 \\
0 & 0 & 1
\end{array}\right\|\left\|\begin{array}{ccc}
-c_{2}^{*} & s_{2}^{*} & 0 \\
-s_{2}^{*} & -c_{2}^{*} & 0 \\
0 & 0 & 0
\end{array}\right\|\|\| \begin{array}{ccc}
1 & 0 & -u^{*} \\
0 & 1 & 0 \\
0 & 0 & 1
\end{array}\|\| \begin{array}{l}
0 \\
0 \\
1
\end{array} \| \\
& +2 \dot{\varphi}_{1}^{*}(*) \dot{\varphi}_{2}^{*}(*)\left\|\begin{array}{ccc}
-1 & 0 & 0 \\
0 & 1 & 0 \\
0 & 0 & 0
\end{array}\right\|\left\|\begin{array} { c c c } 
{ - s _ { 2 } ^ { * } } & { - c _ { 2 } ^ { * } } & { 0 } \\
{ c _ { 2 } ^ { * } } & { - s _ { 2 } ^ { * } } & { 0 } \\
{ 0 } & { 0 } & { 0 }
\end{array} \left|\left\|\mid \begin{array}{l}
0 \\
0 \\
1
\end{array}\right\|\right.\right. \\
& +2 \ddot{\varphi}_{1}^{*}(*) \dot{\mathbf{u}}^{*}(*)\left\|\begin{array}{ccc}
-1 & 0 & 0 \\
0 & -1 & 0 \\
0 & 0 & 0
\end{array}\right\|\left\|\begin{array}{ccc}
c_{2}^{*} & -s_{2}^{*} & r \\
s_{2}^{*} & c_{2}^{*} & 0 \\
0 & 0 & 1
\end{array}\right\|\left\|\left|\begin{array}{ccc}
0 & 0 & -1 \\
0 & 0 & 0 \\
0 & 0 & 0
\end{array}\right|\right\|\left\|\begin{array}{|l}
0 \\
0 \\
1
\end{array}\right\| \\
& +2 \dot{\boldsymbol{\varphi}}_{2}^{*}(*) \dot{\mathbf{u}}^{*}(*)\left\|\begin{array}{ccc}
0 & -1 & 0 \\
1 & 0 & 0 \\
0 & 0 & 1
\end{array}\right\|\left\|\begin{array}{ccc}
-s_{2}^{*} & -c_{2}^{*} & 00 \\
c_{2}^{*} & -s_{2}^{*} & 0 \\
0 & 0 & 0
\end{array}\right\|\|\| \mid \begin{array}{ccc}
0 & 0 & 1 \\
0 & 0 & 0 \\
0 & 0 & 0
\end{array}\|\|\left\|\begin{array}{c}
0 \\
0 \\
1
\end{array}\right\| \|
\end{aligned}
$$

In the developing form the obtained equation will be

$$
\begin{aligned}
& \ddot{\varphi}_{2}^{*}=-\ddot{\varphi}_{1}^{*}+\frac{c_{2}^{*}}{u^{*}} r \ddot{\varphi}_{1}^{*}+\frac{s_{2}^{*}}{u^{*}} \dot{\varphi}_{1}^{* 2}+2\left(\dot{\varphi}_{1}^{*}+\dot{\varphi}_{2}^{*}\right) \frac{\dot{u}^{*}}{u^{*}} \\
& \ddot{u}^{*}=s_{2}^{*} r \ddot{\varphi}_{1}^{*}-u^{*}\left(\dot{\varphi}_{1}^{*}+\dot{\varphi}_{2}^{*}\right)^{2}+r c_{2}^{*} \dot{\varphi}_{1}^{* 2} .
\end{aligned}
$$

The absolute angular velocity of the rod $A C$ and the relative velocity of the rod $A C$ with respect to the turning pistton $\mathrm{B}$ are of the form respectively

$$
\begin{aligned}
& \varepsilon_{2}=\ddot{\varphi}_{1}^{*}+\ddot{\varphi}_{2}^{*}=\frac{c_{2}^{*}}{u^{*}} r \ddot{\varphi}_{1}^{*}+\frac{s_{1}^{*}}{u^{*}} r\left(\dot{\varphi}_{1}^{*}+\dot{\varphi}_{2}^{*}\right)^{2}+2\left(\dot{\varphi}_{1}^{*}+\dot{\varphi}_{2}^{*}\right) \frac{\dot{u}^{*}}{u^{*}} \\
& a_{r}=-\ddot{u}^{*}=s_{2}^{*} r \ddot{\varphi}_{1}^{*}-u^{*}\left(\dot{\varphi}_{1}^{*}+\dot{\varphi}_{2}^{*}\right)^{2}-r c_{2}^{*} \dot{\varphi}_{1}^{* 2} .
\end{aligned}
$$

At the given time we have

$$
\begin{aligned}
& u^{*}=\sqrt{r^{2}+h^{2}} ; \quad c_{2}^{*}=\frac{r}{\sqrt{r^{2}+h^{2}}} ; \\
& s_{2}^{*}=\frac{h}{\sqrt{r^{2}+h^{2}}} ; \quad \dot{u}^{*}=s_{2}^{*} r \dot{\varphi}_{1}^{*} ; \quad \dot{\varphi}_{2}^{*}=-\dot{\varphi}_{1}^{*}+\frac{c_{2}^{*}}{u^{*}} r \dot{\varphi}_{1}^{*} .
\end{aligned}
$$

By substituting these date into the above expressions we find

$$
\begin{aligned}
& \varepsilon_{2}=\frac{r^{2}}{r^{2}+h^{2}} \varepsilon_{1}+\frac{h r^{3}}{\left(r^{2}+h^{2}\right)^{2}}\left[2+\frac{r^{2}}{r^{2}+h^{2}}\right] \omega_{1}^{2} ; \\
& a_{r}=\frac{h r}{\sqrt{r^{2}+h^{2}}} \varepsilon_{1}-\frac{r^{2}}{\sqrt{r^{2}+h^{2}}}\left(\frac{r^{2}}{r^{2}+h^{2}}-1\right) \omega_{1}^{2},
\end{aligned}
$$

where $\omega_{1}$ and $\varepsilon_{1}$ are the absolute angular velocity and acceleration of the crank $O A$ at the given instant of time respectively. 


\section{CONCLUSION}

In the paper the transmission matrix method is applied for investigating the relative motion of planar mechanisms. The proposed method are an useful tool for solving the problems of kinematics and dynamics of rigid body systems, for example, the synthetic and analysic problem of motions. It is important that by such a way it is possible to approach the softwere as MATCAD, MAPLE,... for studing the complexed systems

This work is completed by financial support of the Basic Program in Natural Science.

\section{REFERENCES}

1. Do Dang Khoa, Tran Duc, Dinh Van Phong, Do Sanh, The Optimal Control for the system of program motion, The $3^{\text {rd }}$ Vietnam Conference on Mechatronics, VMC 2006, Hanoi, Vietnam, Publ. Hanoi National University, pp. 87-95

2. Do Sanh, Do Dang Khoa, The Method of Transmission Matrix for Investigating Planar Mechanism,

3. E. J. Haug, Computer Aided Kinematics and Dynamics of Mechanical Systems, Volume I, Basic Method, Alyn\& Bacon, NewYork, 1989.

4. W. Khali and E. Dombre, Modeling, Identification and Control of Robots, London, Hermes, 2002.

5. J. P. Merler, Jacobian, Manipulability, Condition Number and Accurcy of Parallel Robots, Journal of Mechanical Design 128 (1) (2006) 199-206.

6. E. NikravesP, Computer Aided Analysics of Mechanical System, Printice Hall, New York; 1989.

7. B. Paul, Kinematics and Dynamics of Planar Machinery, Printice Hall, NewYork,1989

8. A. Shabana, Dynamics of Multibody Systems, John Willey \&Sonc, NewYork/Chichester/Brisbane, 1989.

Received October 4, 2007

\section{PHƯƠNG PHÁP MA TRẬN TRUYỀn KHẢO SẢT CÁC CHUYỂN ĐộNG TƯƠNG DỐI}

Nội dung của bài báo là tiếp tục xây dựng phương pháp ma trận truyền để khảo sát bài toán chuyển động tương đối cho hệ vật răn (trong bài báo đề cập đến bài toán các chuyển động phẳng). Bài toán như vậy được quan tâm nhiều trong kỹ thuật, đặc biệt trong các bài toán tổng hợp chuyển động, trước hết là những bài toán điều khiển chuyển đông chương trình các hệ cơ học. Một kết quá quan trọng là phương pháp được đề xuất cho một quan điểm tồng quát đối với bài toán khảo sát động học các hệ cơ học. 